\title{
Incidence and Risk Factors of Peripherally Inserted Central Catheter-related Complications in Patients with Different Disease Types
}

\author{
Cao Mingkun ${ }^{1}$, Yin Yuxia ${ }^{1,2}$, Gao Wei ${ }^{3}$, Feng Shengyu ${ }^{4}$, Wang Dengxu ${ }^{4}$, Wan Min ${ }^{5,6}$, \\ Liu Chenghu ${ }^{5,6}$, Wang Luning ${ }^{2}$, Ge Junbo ${ }^{7}$, Zhang Haijun ${ }^{1,8}$, * \\ ${ }^{1}$ National United Engineering Laboratory for Biomedical Material Modification, Dezhou, China \\ ${ }^{2}$ School of Materials Science and Engineering, University of Science and Technology, Beijing, China \\ ${ }^{3}$ PICC Clinic, Qilu Hospital of Shandong University, Jinan, China \\ ${ }^{4}$ National Engineering Technology Research Center for Colloidal Materials \& Key Laboratory of Special Functional Aggregated Materials, \\ Ministry of Education, School of Chemistry and Chemical Engineering, Shandong University, Jinan, China \\ ${ }^{5}$ Shandong Quality Inspection Center for Medical Devices, Jinan, China \\ ${ }^{6}$ Shandong Provincial Key Laboratory of Biological Evaluation of Medical Devices, Jinan, China \\ ${ }^{7}$ Shanghai Institute of Cardiovascular Diseases, Zhongshan Hospital, Fudan University, Shanghai, China \\ ${ }^{8}$ Department of Vascular \& Intervention, Tenth Peoples' Hospital of Tongji University, Shanghai, China
}

Email address:

zhanghaijun@tongji.edu.cn (Zhang Haijun)

${ }^{*}$ Corresponding author

To cite this article:

Cao Mingkun, Yin Yuxia, Gao Wei, Feng Shengyu, Wang Dengxu, Wan Min, Liu Chenghu, Wang Luning, Ge Junbo, Zhang Haijun. Incidence and Risk Factors of Peripherally Inserted Central Catheter-related Complications in Patients with Different Disease Types. European Journal of Preventive Medicine. Vol. 7, No. 6, 2019, pp. 108-111. doi: 10.11648/j.ejpm.20190706.13

Received: September 29, 2019; Accepted: October 31, 2019; Published: November 7, 2019

\begin{abstract}
Background: Cancer patients or critical patients need reliable vascular access to meet the requirements of transporting therapeutic drugs or nutrients. Peripherally inserted central catheters (PICCs) are widely used in these patients, however, the incidence of PICC-related complications is high and the data on specific diseases are not yet clear. Methods: We collected geographic characteristics and insertion information of patients with lung cancer, breast cancer, digestive tract tumor and ICU status who underwent PICC during May 2017 to June 2018. Univariate and multivariate analysis were used to estimate the risk factors of PICC-related complications. Results: 634 lung cancer, 979 breast cancer, 1453 digestive tract cancer, and 374 ICU status patients were enrolled in the study with $351(10.2 \%)$ developed complications. There was no difference in the distribution of complications among different disease types ( $\mathrm{P}>0.05)$. Drinking (OR 2.15, 95 CI\% 1.05-3.69, $P=0.012$ ) and prior surgery (OR $1.97,95 \%$ CI $1.05-3.69, \mathrm{P}=0.035$ ) were risk factors of breast cancer patients. Prior surgery (OR 2.51, 95\% CI 1.54-4.09, P<0.001) and site of PICC (OR 1.56, 95\% CI 1.10-2.23, $\mathrm{P}=0.014$ ) were the influenced factors of digestive tract tumor patients. Complications of ICU status patients were influenced by smoke (OR 5.83, 95\% CI 1.30-26.14, $\mathrm{P}=0.021$ ) and prior surgery (OR 3.43, 95\% CI 1.44-8.13, $\mathrm{P}=0.005$ ). Conclusion: The targeted and reasonable prevention and nursing care should be focused on by medical staff.
\end{abstract}

Keywords: Peripherally Inserted Central Catheters, Disease Type, Complications, Risk Factors

\section{Introduction}

PICC has been widely used in cancer and critically ill patients for some advantages such as convenient insertion, short procedure time, reliable efficacy and fewer complications compared to central venous catheter $[1,2]$. Despite the above advantages, PICC-related complications were still at an increased risk [3-5]. The evidence on prevent measures to PICC-related complications is urgently-needed. 
Although previous studies have reported risk factors for complications, no consensus has been reached so far. According to the World Health Organization (WHO), Lung, colorectal, stomach and liver cancer are the most common types of cancer in men, while breast, colorectal and lung cancer are the most common among women [6]. In this study, we selected three common cancers (lung cancer, breast cancer and digestive tract tumor) and ICU status patients to explore their PICC-related complications and risk factors aiming to formulate targeted and reasonable prevention and nursing care for patients with specific disease types.

\section{Materials and Methods}

\subsection{Design}

This study was performed as a retrospective study at multicenter. All PICCs were 4F, silicone catheters and were placed by the professional nurse team according to instructions. The PICCs used in this study were a subset of Cathicoid ${ }^{\mathrm{TM}}$ PICCs produced by Branden Medical Scientific, Inc. From May 2017 to June 2018, patients with lung cancer, breast cancer, digestive tract tumor and ICU status who underwent PICC placement were eligible for inclusion in this study. We collected data on demographic characteristics, insertion situation and complication information during insertion and follow-up period. All data was provided by professional nurses teams who were responsible for catheterization and follow-up of patients included in the study.

\subsection{PICC Placement Technique}

All PICCs insertion followed care and maintenance protocols formulated by each center. Standard aseptic precaution including hand hygiene, barrier precautions and chlorhexidine skin antisepsis was performed during insertion.
Portable ultrasonography was used to evaluate insertion vein before insertion and catheter tip location was confirmed by chest radiography. Catheters were routinely flushed by nurses. The primary study endpoints were incidence of PICC-related complications. All patients were followed-up until they met criteria for a PICC-related complication or PICC removal.

\subsection{Statistical Analysis}

Categorical variables were reported as frequency (percent) and continuous variables as means ( \pm standard deviation $[\mathrm{SD}]$ ). Baseline comparisons of different disease types were performed using the chi-square test or Fisher's exact test for categorical variables. One-way ANOVA was used to compare difference for continuous variables. Comparisons of complications in different diseases used Cochran-Mantel-Haenszel (CMH) test. Univariate and multivariate analysis were used to explore risk factors of complications in different disease types. Enter method was used for logistic regression models. $\alpha=0.05$ was used for the significance to enter the model, and $\alpha=0.10$ was used for significance level to delete from the model. A two-sided $P$ value of less than 0.05 was considered to indicate statistical significance. All analyses were performed with the SPSS software (version 24.0, SPSS Inc., IBM, NY, USA).

\section{Results}

Totally 634 lung cancer, 979 breast cancer, 1453 digestive tract cancer, and 374 ICU status patients were enrolled in the study with $351(10.2 \%)$ developed complications. No significant difference was observed in most basic characteristics $(\mathrm{P}>0.05)$ expect for gender $(\mathrm{P}=0.014)$ and vein of PICC $(\mathrm{P}<0.001)$. The specific results of other basic variables were showed in table 1 .

Table 1. Baseline characteristics of patients.

\begin{tabular}{|c|c|c|c|c|c|}
\hline Characteristics & Lung cancer $N=634$ & Breast cancer $\mathrm{N}=979$ & Digestive tract tumor $N=1453$ & ICU status $\mathrm{N}=374$ & Pvalue \\
\hline Age*, year & $55.3 \pm 12.5$ & $54.8 \pm 12.8$ & $54.3 \pm 12.6$ & $53.8 \pm 12.6$ & 0.187 \\
\hline Gender, Male & $28(45.4)$ & $40(41.4)$ & $69(47.8)$ & $15(42.5)$ & 0.014 \\
\hline Weight*, kg & $59.7 \pm 12.2$ & $61.1 \pm 19.8$ & $61.2 \pm 13.9$ & $60.6 \pm 16.2$ & 0.197 \\
\hline Marriage, married & $50(\overline{79} .7)$ & $78(80.5)$ & $117(80.9)$ & $31(82.9)$ & 0.649 \\
\hline Smoke & $6(10.4)$ & $9(9.3)$ & $12(8.6)$ & $2(6.4)$ & 0.174 \\
\hline Drinking & $5(7.9)$ & $8(8.2)$ & $10(7.2)$ & $2(6.4)$ & 0.670 \\
\hline Coronary heart disease $^{\Delta}$ & $1(1.6)$ & $1(1.1)$ & $3(2.1)$ & (1.6) & 0.359 \\
\hline Diabetic & $15(2.4)$ & $1(1.8)$ & $2(1.9)$ & $1(2.7)$ & 0.716 \\
\hline Liver cirrhosis $^{\Delta}$ & $(0.9)$ & $4(0.4)$ & $1(1.2)$ & $(0.5)$ & 0.215 \\
\hline Prior surgery & $5(9.1)$ & $7(7.5)$ & $12(8.5)$ & $3(8.6)$ & 0.651 \\
\hline Site of PICC ${ }^{\Delta \Delta}$ insertion $^{\Delta}$ & & & & & 0.062 \\
\hline Right arm & $30(48.1)$ & $50(51.5)$ & $78(54.0)$ & $18(49.7)$ & \\
\hline Left arm & $32(51.7)$ & $47(48.5)$ & $66(46.0)$ & $18(50.3)$ & \\
\hline Vein of $\mathrm{PICC}^{\Delta}$ insertion ${ }^{\Delta}$ & & & & & $<0.001$ \\
\hline Basilic vein & $55(86.9)$ & $85(87.5)$ & $123(85.1)$ & $32(86.9)$ & \\
\hline Brachial vein & $3(5.7)$ & $6(6.3)$ & $11(7.6)$ & $2(7.5)$ & \\
\hline Median vein & $3(5.4)$ & $3(4.0)$ & $7(5.3)$ & $1(3.7)$ & \\
\hline Cephalic vein & $1(1.9)$ & $2(2.1)$ & $2(2.0)$ & (1.9) & \\
\hline Other veins & $(0.2)$ & $(0.0)$ & $(0.1)$ & $(0.0)$ & \\
\hline Inserting Length*, mm & $41.3 \pm 4.2$ & $41.0 \pm 4.2$ & $41.2 \pm 4.2$ & $40.9 \pm 4.6$ & 0.501 \\
\hline
\end{tabular}

${ }^{*}:$ t test; ${ }^{\Delta}$ : Fisher exact test 
There was no difference in the incidence of complications among different disease types $(\mathrm{P}>0.05)$. The incidence of complications were $9.3 \%, 10.8 \%, 9.6 \%$ and $12.6 \%$ for lung cancer, breast cancer, digestive tract tumor, and ICU status patients. OR and 95\% CI were showed in table 2 .

Table 2. Complications of patients with different disease types.

\begin{tabular}{|c|c|c|c|c|c|}
\hline Disease types & Patients with complications $(\mathrm{N}=351,10.2)$ & Patients without complications $(\mathrm{N}=3089,89.8)$ & OR & $95 \% \mathrm{CI}$ & P value \\
\hline Lung cancer & $5(9.3)$ & $57(90.7)$ & 0.88 & $0.66-1.19$ & 0.409 \\
\hline Breast cancer & $10(10.8)$ & $87(89.2)$ & 1.10 & $0.86-1.40$ & 0.446 \\
\hline Digestive tract tumor & $13(9.6)$ & $131(90.4)$ & 0.89 & $0.71-1.11$ & 0.291 \\
\hline
\end{tabular}

No risk factor was found for complications of lung cancer patients. In univariate analysis, drinking and prior surgery were related to complications of breast cancer patients, smoke, drinking, prior surgery and site of PICC were related to complications of digestive tract tumor patients, smoke, drinking, diabetic, and prior surgery were related to complications of ICU status patients. In multivariate analysis, drinking (OR 2.15, $95 \mathrm{CI} \%$ 1.05-3.69, $\mathrm{P}=0.012$ ) and prior surgery (OR 1.97, 95\% CI 1.05-3.69, $\mathrm{P}=0.035)$ were risk factors of breast cancer patients. Prior surgery (OR 2.51, 95\% CI 1.54-4.09, $\mathrm{P}<0.001)$ and site of PICC (OR 1.56, 95\% CI $1.10-2.23, \mathrm{P}=0.014$ ) were the influenced factors of digestive tract tumor patients. Complications of ICU status patients were influenced by smoke (OR 5.83, 95\% CI 1.30-26.14, $\mathrm{P}=0.021)$ and prior surgery (OR 3.43, 95\% CI 1.44-8.13, $\mathrm{P}=0.005)$.

Table 3. Univariable and multivariable analysis for risk factors of complicatins.

\begin{tabular}{|c|c|c|c|c|c|}
\hline & \multicolumn{2}{|c|}{ Univariable analysis } & \multicolumn{3}{|c|}{ Multivariable analysis } \\
\hline & $\chi^{2}$ & P value & OR & $95 \% \mathrm{CI}$ & P value \\
\hline \multicolumn{6}{|l|}{ Breast cancer } \\
\hline Drinking & 9.80 & 0.002 & 2.15 & $1.18-3.9$ & 0.012 \\
\hline Prior surgery & 7.72 & 0.005 & 1.97 & $1.05-3.69$ & 0.035 \\
\hline \multicolumn{6}{|c|}{ Digestive tract tumor } \\
\hline Smoke & 6.54 & 0.011 & 1.56 & $0.69-3.54$ & 0.287 \\
\hline Drinking & 5.74 & 0.017 & 1.11 & $0.46-2.68$ & 0.819 \\
\hline Site of PICC & 7.21 & 0.007 & 1.56 & $1.10-2.23$ & 0.014 \\
\hline \multicolumn{6}{|l|}{ ICU status } \\
\hline Smoke $^{\Delta}$ & - & 0.001 & 5.83 & $1.30-26.14$ & 0.021 \\
\hline Drinking $^{\Delta}$ & - & 0.020 & 0.67 & $0.13-3.44$ & 0.633 \\
\hline Diabetic $^{\Delta}$ & - & 0.026 & 3.59 & $0.84-15.32$ & 0.084 \\
\hline Prior surgery ${ }^{\Delta}$ & - & 0.001 & 3.43 & $1.44-8.13$ & 0.005 \\
\hline
\end{tabular}

${ }^{\Delta}$ : Fisher exact test

\section{Discussion}

In this study, we explored the incidence and risk factors of PICC-related complications in different disease types. The main findings were as follows: 1) The total incidence of complications in lung cancer, breast cancer, digestive tract tumor and ICU status patients was $10.2 \%$; 2) The incidence of complications were similar among the above four disease types; 3) Drinking and prior surgery were the most common risk factors for PICC-related complications.

Previous studies have reported the incidence of PICCrelated complications in cancer patients patients [3, 7-9]. Bertoglio S. et al reported a $24.7 \%$ incidence of PICC-related complications [3]. Yap YS. et al showed a $15.9 \%$ rate of the overall complication ${ }^{9}$. But their research subjects were all patients with different types of tumors and did not explore the incidence of complications of a specific disease. Lung cancer, breast cancer and digestive tract tumor were common among cancer patients and accounted for a large proportion of catheter patients. To study the incidence and risk factors of complications in these patients can provide reference for nursing staff to take targeted nursing measures. In our study, the PICC-related complications incidence of lung cancer, breast cancer, digestive tract tumor and ICU status were $9.3 \%, 10.8 \%, 9.6 \%$ and $12.6 \%$ respectively and significantly lower than some reports [10-12]. The lower incidence may be related to the material of PICC and the operation of medical staff. Although the incidence of complications in patients with different disease types was different, there was no statistical significance.

For lung cancer patients, we did not find risk factors of PICC-related complications. Prior surgery was the risk factor of PICC-related complication for breast cancer, digestive tract tumor and ICU status patients. For cancer patients and ICU patients, they may have received surgery just before catheterization, which has a certain impact on their physiological and psychological conditions. So their antiinfection and other abilities will decline which creat conditions for the occurrence of complications. For breast cancer patients, drinking was risk factor of PICC-related complications. Alcohol may affect the health of blood vessel $[13,14]$ and the performance of catheters [15]. Left arm insertion affected the incidence of PICC-related complications in digestive tract tumor patients. Compared 
with the right arm, the left arm usually has less exercise and its vascular performance may be inferior to that of the right arm, so it is more likely to have complications. Smoke ICU patients were more likely to occur complication. It may due to their poor lung function and vascular status caused by smoking.

The greatest significance of this study is to make the medical staff focus on the key care of different diseases. Despite the fact that we've done research that no one else has done on the incidence and risk factors of specific diseases, there are several limitations of this study. Firstly, we have not made any further exploration for each specific complication. Secondly, the factors influencing the incidence of complications are not comprehensive. These limitation is worthwhile for us to overcome and do further research.

\section{Conclusion}

The incidence of PICC-related complications was $10.2 \%$ in lung cancer, breast cancer, digestive tract cancer, and ICU status patients and the incidence of complications were $9.3 \%$, $10.8 \%, 9.6 \%$ and $12.6 \%$ respectively. Prior surgery was the risk factor of PICC-related complication. To reduce complications, we also should pay attention to drinking breast cancer patients, Left arm insertion digestive tract tumor patients and smoke ICU patients except patients with prior surgery. For other patients we did not focus on in this study may take targeted and reasonable nursing measures with reference to these conclusions.

\section{References}

[1] Cotogni P, Barbero C, Garrino C, et al. Peripherally inserted central catheters in non-hospitalized cancer patients: 5-year results of a prospective study. Supportive care in cancer: official journal of the Multinational Association of Supportive Care in Cancer. Feb 2015; 23 (2): 403-409.

[2] Clemence BJ, Maneval RE. Risk factors associated with catheter-related upper extremity deep vein thrombosis in patients with peripherally inserted central venous catheters: literature review: part 1. Journal of infusion nursing: the official publication of the Infusion Nurses Society. May-Jun 2014; 37 (3): 187-196.

[3] Bertoglio S, Faccini B, Lalli L, Cafiero F, Bruzzi P. Peripherally inserted central catheters (PICCs) in cancer patients under chemotherapy: A prospective study on the incidence of complications and overall failures. Journal of surgical oncology. May 2016; 113 (6): 708-714.
[4] Franklin I, Gilmore C. Placement of a peripherally inserted central catheter into the azygous vein. Journal of medical radiation sciences. Jun 2015; 62 (2): 160-162.

[5] Wang W, Zhao C, Ji Q, Liu Y, Shen G, Wei L. Prevention of peripherally inserted central line-associated blood stream infections in very low-birth-weight infants by using a central line bundle guideline with a standard checklist: a case control study. BMC pediatrics. Jun 18 2015; 15: 69.

[6] Organization WH. Cancer. Available at: https://www.who.int/health-topics/cancer\#tab=tab_1.

[7] Dutia M, White RH, Wun T. Risk assessment models for cancer-associated venous thromboembolism. Cancer. Jul 15 2012; 118 (14): 3468-3476.

[8] Saber W, Moua T, Williams EC, et al. Risk factors for catheter-related thrombosis (CRT) in cancer patients: a patient-level data (IPD) meta-analysis of clinical trials and prospective studies. Journal of thrombosis and haemostasis: JTH. Feb 2011; 9 (2): 312-319.

[9] Yap YS, Karapetis C, Lerose S, Iyer S, Koczwara B. Reducing the risk of peripherally inserted central catheter line complications in the oncology setting. European journal of cancer care. Sep 2006; 15 (4): 342-347.

[10] Scrivens N, Sabri E, Bredeson C, McDiarmid S. Comparison of complication rates and incidences associated with different peripherally inserted central catheters (PICC) in patients with hematological malignancies: a retrospective cohort study. Leukemia \& lymphoma. Aug 7 2019: 1-9.

[11] Kang J, Chen W, Sun W, et al. Peripherally inserted central catheter-related complications in cancer patients: a prospective study of over 50, 000 catheter days. J Vasc Access. Mar 21 2017; 18 (2): 153-157.

[12] Paje D, Conlon A, Kaatz S, et al. Patterns and Predictors of Short-Term Peripherally Inserted Central Catheter Use: A Multicenter Prospective Cohort Study. Journal of hospital medicine. Feb 2018; 13 (2): 76-82.

[13] de Zambotti M, Willoughby AR, Baker FC, Sugarbaker DS, Colrain IM. Cardiac autonomic function during sleep: effects of alcohol dependence and evidence of partial recovery with abstinence. Alcohol. Jun 2015; 49 (4): 409-415.

[14] Gardner JD, Mouton AJ. Alcohol effects on cardiac function. Comprehensive Physiology. Apr 2015; 5 (2): 791-802.

[15] Crnich CJ, Halfmann JA, Crone WC, Maki DG. The effects of prolonged ethanol exposure on the mechanical properties of polyurethane and silicone catheters used for intravascular access. Infection control and hospital epidemiology. Aug 2005; 26 (8): 708-714. 